\title{
Scalar Quantum Electrodynamics on the Lattice as Classical Statistical Mechanics
}

\author{
Gian Fabrizio De Angelis, Diego de Falco, and Francesco Guerra \\ Institute of Physics`, University of Salerno, Salerno, Italy
}

\begin{abstract}
Wilson's lattice approximation allows us to apply classical statistical mechanics ideas to the study of Scalar Quantum Electrodynamics. Our main tools are Griffiths-Kelly-Sherman inequalities, the transfer matrix formalism and exponential bounds. Our main result is the existence of the infinite volume limit for every value of the coupling parameters.
\end{abstract}

\section{Introduction and Outline}

In 1974 Wilson [18] suggested the interesting possibility of performing the YangMills [19] step of promoting a global symmetry to a local one after introducing a lattice ultraviolet cutoff on the globally invariant theory. This procedure not only gave a very convenient gauge invariant prescription for the ultraviolet regularization of gauge theories, but also, due to the use of a Euclidean space-time lattice, as compared to the space lattice-continuous time of the alternative Hamiltonian approach [10], opened the possibility of using classical statistical mechanics methods for the study of quantum gauge fields in the spirit of the general program of Quantum Field Theory as Classical Statistical Mechanics [5, 9, 13]. An analysis of the structure of the statistical mechanical models thus introduced and a preliminary exploration by mean field techniques of their properties was given in [1]. An extensive rigorous discussion, in the strong coupling regime, of the thermodynamic limit and of the existence of a mass gap was given in $[14,15]$. Important upper bounds holding for any value of the coupling constant for the pure $U(1)$ model were given in [6]; a very promising step towards the full understanding of the critical behavior of the same model is in the analysis of [7] based on a mathematically rigorous examination of the role of Polyakov's instantons [16]: this method suggests that there is no mass generation at sufficiently low coupling for $d \geqq 4$, as opposed to the $d=3$ case.

This paper is devoted to the study of Wilson's version of Scalar Quantum Electrodynamics on the lattice briefly described in Section 2. In Section 3 we discuss

* Postal address: Istituto di Fisica dell' Universitá, Via Vernieri 42, I-84100 Salerno, Italy 
GKS inequalities for this model. The resulting statements of monotonicity in the cutoff volume must be supplemented by suitable upper bounds in order to insure the existence and non triviality of the thermodynamic limit; these bounds are discussed in Section 5 in which use is made of the transfer matrix formalism introduced in Section 4

\section{The Model}

We consider a two component Euclidean field $\varphi(n)=\left(\varphi_{1}(n), \varphi_{2}(n)\right) \in \mathbb{R}^{2}$ self interacting through a quartic interaction and coupled with a $U(1)$ gauge field on the space-time lattice $\varepsilon \mathbb{Z}^{d}, \varepsilon>0$.

It is convenient to introduce polar coordinates by

$$
\varphi_{1}(n)=\varrho(n) \cos \theta(n), \quad \varphi_{2}(n)=\varrho(n) \sin \theta(n), \quad \varrho(n) \geqq 0, \quad-\pi \leqq \theta(n) \leqq \pi .
$$

The Euclidean action in the cutoff volume $\Lambda$ is then given by:

$$
\begin{aligned}
S_{\Lambda}= & \sum_{n \in \Lambda} P(\varrho(n))-\beta_{l} \sum_{n, n^{\prime} \in A} \varrho(n) \varrho\left(n^{\prime}\right) \cos \left(\theta(n)-\theta\left(n^{\prime}\right)-A\left(n, n^{\prime}\right)\right) \\
& +-\underset{p}{ } \beta_{\mathfrak{p} \subset A} \sum \cos (\partial A(\mathfrak{P})) .
\end{aligned}
$$

Here $n, n^{\prime}$ is a couple of nearest neighbor sites, $A\left(n, n^{\prime}\right)=-A\left(n^{\prime}, n\right)$ is an angle variable associated to the ordered link $n, n^{\prime} n<n^{\prime}, P(\varrho)$ is an even fourth degree polynomial bounded below and $\beta_{l}, \beta_{p}$ are suitable non negative constants. The sum $\sum_{\mathfrak{P} \subset A}$ extends to all "plaquettes" (elementary squares on the lattice) in $\Lambda$ and if $n_{1}, n_{2}$, $n_{3}, n_{4}$ are the four consecutive vertices of the plaquette $\mathfrak{P}, \partial A(\mathfrak{P})=A\left(n_{1}, n_{2}\right)$ $+A\left(n_{2}, n_{3}\right)+A\left(n_{3}, n_{4}\right)+A\left(n_{4}, n_{1}\right)$.

The averages $\langle\cdot\rangle$ of functions of the fields are taken with respect to the normalized measure:

$$
d \mu_{\Lambda}=Z_{\Lambda}^{-1} \exp \left\{-U_{\Lambda}\right\} d \omega_{\Lambda}(\varrho) d \theta_{\Lambda} d A_{\Lambda}=Z_{\Lambda}^{-1} \exp \left\{-U_{\Lambda}\right\} d \sigma_{\Lambda},
$$

where $d \theta_{\Lambda}, d A_{\Lambda}$ are the normalized product measures for the variables $\theta, A, d \omega_{\Lambda}(\varrho)$ $=\prod_{n \in \Lambda} d \omega(\varrho(n))$ with

$$
d \omega(\varrho)=\left[\int_{\mathbb{R}_{+}} \exp \{-P(\varrho)\} \varrho d \varrho\right]^{-1} \exp \{-P(\varrho)\} \varrho d \varrho
$$

and $U_{\Lambda}$ is defined as $U_{\Lambda}=S_{\Lambda}-\sum_{n \in \Lambda} P(\varrho(n))$.

This measure is invariant under local gauge transformations

$$
\theta(n) \rightarrow \theta(n)+\chi(n), \quad A\left(n, n^{\prime}\right) \rightarrow A\left(n, n^{\prime}\right)-\chi\left(n^{\prime}\right)+\chi(n) .
$$

We will refer to the boundary conditions considered up to now as to "free" boundary conditions. In the following we will consider also periodic $(P)$ boundary conditions corresponding to the choice of a parallelepiped as cutoff volume with the 
convention of considering as nearest neighbors corresponding points on opposite faces. Corresponding to the links added in this way there will be new link variables $A$ and new link and plaquette terms in the action. As a final remark, we observe that, as we do not commit ourselves to a particular choice of the sign of the quadratic term in $P(\varrho)$, the considerations which follow apply to the lattice version of the Higgs model as well as to Scalar Quantum Electrodynamics.

\section{Griffiths-Kelly-Sherman Inequalities}

Our starting point is the following result of Ginibre's analysis of GKS inequalities [4]:

Lemma 3.1. Let $K$ be the direct product of a finite number of copies of the circle group $T$, let $d \sigma$ be the normalized Haar measure on $K$. Then for every $n$ and for every choice $f_{1}, f_{2}, \ldots, f_{n}$ of functions in the multiplicative convex cone generated by the set $S$ of the real parts of the characters of $K$, the following inequalities hold:

$$
\int d \sigma(x) d \sigma\left(x^{\prime}\right) \prod_{i=1}^{n}\left(f_{i}(x) \pm f_{i}\left(x^{\prime}\right)\right) \geqq 0
$$

from which GKS inequalities follow.

It was observed in [2] that, at least in the case when the scalar field variables are restricted to range on the unit circle, our model falls under the hypotheses of Lemma 3.1 and it was therefore possible to prove the existence of the thermodynamic limit.

Now we wish to extend the GKS inequalities apparatus to the full model of Section 2.

For any subset $M$ in $\varepsilon \mathbb{Z}^{d}$ call $L(M)$ the set of links in $M$ and set $K_{\Lambda}=\underset{n \in A}{X} \underset{l \in L(\Lambda)}{X} T$; call $S_{\Lambda}$ the set of the real parts of the characters of $K_{\Lambda}, R_{A}$ the set of monomials

$$
\prod_{n \in \Lambda}[\varrho(n)]^{p(n)}
$$

with $p(n) \in\{0,1\}, Q_{A}$ the set of polynomials of elements of $S_{\Lambda}$ and $R_{A}$ with non negative coefficients and $H_{\Lambda}$ the collection of functions of the form

$$
\sum_{n, n^{\prime} \in \Lambda} a\left(n, n^{\prime}\right) \varrho(n) \varrho\left(n^{\prime}\right) \cos \left(\theta(n)-\theta\left(n^{\prime}\right)-A\left(n, n^{\prime}\right)\right)+\sum_{\mathfrak{P} \subset A} b(\mathfrak{P}) \cos (\partial A(\mathfrak{P}))+\sum_{n \in A} c(n) \varrho(n)
$$

with non negative coefficients $a, b, c$, where the sum extends to sites, links and plaquettes in $\Lambda$; then:

Theorem 3.2. For $h \in H_{A}, Z_{h}=\int d \sigma_{A} \exp \{h\},\langle f\rangle_{h}=Z_{h}^{-1} \int d \sigma_{A} f \exp \{h\}$ the following inequalities hold:
i) $Z_{h} \geqq 1$.
ii) $\langle f\rangle_{h} \geqq 0$.
iii) $\langle f g\rangle_{h} \geqq\langle f\rangle_{h}\langle g\rangle_{h}$ for any $f, g \in Q_{\Lambda}$.
iv) $Z_{h_{1}+h_{2}} \geqq Z_{h_{1}} \times Z_{h_{2}}$.
v) $\left\langle f \exp h_{1}\right\rangle_{h_{2}} \geqq\langle f\rangle_{h_{2}}\left\langle\exp h_{1}\right\rangle_{h_{2}}$ for $h_{1}, h_{2} \in H_{\Lambda}$ and any $f \in Q_{\Lambda}$. 
Proof. Use the elementary inequality

$$
\int_{\mathbb{R}_{+} \times \mathbb{R}_{+}} d \omega(\varrho) d \omega\left(\varrho^{\prime}\right) \prod_{i=1}^{n}\left(\varrho^{r_{i}} \pm \varrho^{\prime r_{i}}\right) \geqq 0, \quad r_{i} \in\{0,1\},
$$

Lemma 3.1 and classical arguments of Ginibre [4].

Corollary 3.3. If $h_{1}, h_{2} \in H_{\Lambda}$, then $\langle f\rangle_{h_{1}+h_{2}} \geqq\langle f\rangle_{h_{1}}$ for any $f \in Q_{\Lambda}$.

From Theorem 3.2 and its Corollary we derive, in particular, the inequalities $Z_{\Lambda} \geqq 1,\langle f\rangle_{\Lambda} \geqq 0,\langle f g\rangle_{\Lambda} \geqq\langle f\rangle_{\Lambda}\langle g\rangle_{\Lambda}$ for any $f, g \in Q_{\Lambda}$, and the conclusion that, for free boundary conditions, $\langle f\rangle_{\Lambda}$ is monotonically increasing with $\Lambda$ for any $f \in Q_{\Lambda}$.

We also remark that $\langle f\rangle_{A} \leqq\langle f\rangle_{\Lambda}^{(P)} \forall f \in Q_{\Lambda}$, where $\langle\cdot\rangle_{A}^{(P)}$ refers to the expectation with periodic boundary conditions.

Our first result concerning the thermodynamic limit refers to the existence of the pressure:

Theorem 3.4. Set $U_{\Lambda}(r)=U_{\Lambda}-r \sum_{n \in \Lambda} \varrho(n)$ with $r \geqq 0, Z_{\Lambda}(r)=\int d \sigma_{\Lambda} \exp \left\{-U_{\Lambda}(r)\right\}$ and $\alpha_{\Lambda}(r)=|\Lambda|^{-1} \log Z_{\Lambda}(r)$. The limit $\alpha_{\infty}(r)=\lim _{\Lambda \ngtr \mathbb{Z}^{d}} \alpha_{\Lambda}(r)$ exists finite.

Proof. By Theorem 3.2, Proposition iv), $\alpha_{\Lambda}(r)$ is monotone increasing with $\Lambda$ and we need only an upper bound. Now

$$
\begin{aligned}
\exp \left\{-U_{\Lambda}(r)\right\} & \leqq \exp \left\{\beta_{p} n_{\mathfrak{P}}(\Lambda)+r \sum_{n \in \Lambda} \varrho(n)+\beta_{l} \sum_{n, n^{\prime} \in \Lambda} \varrho(n) \varrho\left(n^{\prime}\right)\right\} \\
& \leqq \exp \left\{\beta_{p} n_{\mathfrak{P}}(\Lambda)+r \sum_{n \in \Lambda} \varrho(n)+d \beta_{l} \sum_{n \in \Lambda} \varrho(n)^{2}\right\}
\end{aligned}
$$

where $n_{\mathfrak{P}}(\Lambda) \equiv O(|\Lambda|)$ is the number of plaquettes in $\Lambda$.

Then:

$$
\alpha_{\Lambda}(r) \leqq \text { const. } \times \beta_{p}+\log \int_{\mathbb{R}_{+}} d \omega(\varrho) \exp \left\{r \varrho+\beta_{l} d \varrho^{2}\right\}
$$

\section{The Transfer Matrix}

We choose some direction, say direction 1 , in $\varepsilon \mathbb{Z}^{d}$ to be called the direction of transfer $t$. For the sake of definiteness we take the cutoff region $\Lambda$ to be of the form:

$$
\Lambda=\left[-\varepsilon L_{1}, \varepsilon L_{1}\right] \times \tilde{\Lambda}, \tilde{\Lambda}=\left[-\varepsilon L_{2}, \varepsilon L_{2}\right] \times \ldots \times\left[-\varepsilon L_{d}, \varepsilon L_{d}\right], \quad L_{i} \in \mathbb{N} .
$$

Call $q=\left\{\boldsymbol{\varphi}(n), A\left(n, \dot{n}^{\prime}\right)\right\}_{n, n^{\prime} \in \tilde{A}} \equiv\left\{\varrho(n), \theta(n), A\left(n, n^{\prime}\right)\right\}_{n, n^{\prime} \tilde{\Lambda}}$ a generic configuration of the system at "time" 0 , with the convention of identifying $\tilde{\Lambda}$ with the intersection of $\Lambda$ with the $x_{1}=0$ plane, and call $Q$ the space of such configurations.

The partition function $Z_{\Lambda}^{(P)}$ in a periodic box can be written, within an inessential constant normalization coefficient, as :

$$
Z_{\Lambda}^{(P)}=\int \prod_{i=-L_{1}}^{L_{1}} T\left(q_{i}, q_{i+1}\right) d Q_{i},
$$


where

$$
d Q=\prod_{n \in \tilde{A}} d^{2} \varphi(n) \prod_{\substack{n, n^{\prime} \in \tilde{A} \\ n<n^{\prime}}} \frac{d A\left(n, n^{\prime}\right)}{2 \pi}
$$

and

$$
T\left(q, q^{\prime}\right)=F(q) I\left(q, q^{\prime}\right) F\left(q^{\prime}\right)
$$

with

$$
\begin{aligned}
F(q)= & \exp \left\{-\frac{1}{4} \sum_{n \in \tilde{\Lambda}} \mathrm{P}(\varrho(\mathrm{n}))+\frac{\beta_{l}}{2} \sum_{n, n^{\prime} \in \tilde{A}} \varrho(\mathrm{n}) \varrho\left(\mathrm{n}^{\prime}\right) \cos \left(\theta(n)-\theta\left(n^{\prime}\right)-A\left(n, n^{\prime}\right)\right)\right. \\
& \left.+\frac{\beta_{p}}{2} \sum_{\mathfrak{P} \subset \tilde{A}} \cos (\partial A(\mathfrak{H}))\right\}
\end{aligned}
$$

and

$$
\begin{aligned}
& I\left(q, q^{\prime}\right)=\exp \left\{-\frac{1}{4} \sum_{n \in \tilde{A}} P(\varrho(n))\right\} \\
& \times\left[\int \prod _ { \substack { n \in \tilde { X } \\
[ - \pi , \pi ] ^ { | \tilde { A } } ] } } \frac { d x ( n ) } { 2 \pi } \operatorname { e x p } \left\{\beta_{l} \sum_{n \in \tilde{A}} \varrho(n) \varrho^{\prime}(n) \cos \left(\theta(n)-\theta^{\prime}(n)-x(n)\right)\right.\right. \\
& \left.+\beta_{p} \sum_{\substack{n \in \tilde{A} \\
\underline{m}}} \cos \left(A(n, n+\underline{m})-A^{\prime}(n, n+\underline{m})+x(n+\underline{m})-x(n)\right\}\right] \\
& \cdot \exp \left\{-\frac{1}{4} \sum_{n \in \tilde{T}} P\left(\varrho^{\prime}(n)\right)\right\}
\end{aligned}
$$

(here $\underline{m}$ ranges in the set of primitive translations of $\varepsilon \mathbb{Z}^{d-1}$ ).

We are interested in the integral operators $T$ and $I$ with kernels $T(\cdot, \cdot)$ and $I(\cdot, \cdot)$ on the Hilbert space $\mathscr{H}=L^{2}(Q, d Q)$. Twill be called the transfer matrix of the model.

Lemma 4.1. $T$ is a bounded, selfadjoint, positivity improving operator on $\mathscr{H}$. In particular all the consequences of the Simon-Perron-Frobenius theorem [17] hold for $T$.

Proof. $T(\cdot, \cdot)$ is symmetric [just change $x(n)$ into $-x(n)$ and use the parity of the cosine function]; moreover it is a Hilbert-Schmidt kernel, so that $T$ is bounded and compact on $\mathscr{H}$. The positivity improving character of $T$ follows from the observation that $T\left(q, q^{\prime}\right)>0$.

Notice that the same conclusions hold for the operator $I$.

In the following Section we will be interested, more generally, in perturbed forms of the transfer matrix, namely in operators $T\left(F_{1}, F_{2}\right)$ with kernel $\widehat{F_{1}(q)} I\left(q, q^{\prime}\right) F_{2}\left(q^{\prime}\right)$, where $F_{1}$ and $F_{2}$ are continuous functions in $\mathscr{H}$. We also define $T(F)=T(F, F)$ and call $T_{F}(\cdot, \cdot)$ the kernel of such an operator.

Lemma 4.2. For any continuous $F \in \mathscr{H}, T_{F}(\cdot, \cdot)$ is a continuous non negative Hermitian kernel (8) with

$$
\int T_{F}(q, q) d Q<+\infty .
$$


Proof. Let $b=\sup _{q \in Q} I(q, q)$, then $\int T_{F}(q, q) q Q \leqq b\|F\|_{2}^{2}<+\infty$. Continuity and Hermiticity are evident; we need only check that for any continuous $f$ with compact support

$$
\int \overline{f(q)} I\left(q, q^{\prime}\right) f\left(q^{\prime}\right) \geqq 0 .
$$

Now, by expanding

$$
\exp \left\{\beta_{l} \sum_{n \in \tilde{\Lambda}} \varrho(\mathrm{n}) \varrho^{\prime}(\mathrm{n}) \cos \left(\theta(n)-\theta^{\prime}(n)-x(n)\right)\right\}
$$

in a power series and by performing the $\varrho$ integrations first, the integral $\int \bar{f}(q) I\left(q, q^{\prime}\right) f\left(q^{\prime}\right)$ can be cast into the form

$$
\sum_{K} \int d \underline{\alpha} d \underline{\alpha}^{\prime} \overline{\psi_{K}(\underline{\alpha})} h_{K}\left(\underline{\alpha}-\underline{\alpha}^{\prime}\right) \psi_{K}\left(\underline{\alpha}^{\prime}\right)
$$

where $\underline{\alpha}$ and $\underline{\alpha}^{\prime}$ are the angular parts of $q$ and $q^{\prime}$ and $h_{K}$, by GKS inequalities, is a function of positive type. By a limiting argument it then follows that $\langle\psi, T(F) \psi\rangle \geqq 0$ for every $\psi \in \mathscr{H}$.

By standard arguments [8] one can conclude that $T(F)$ is trace class for every continuous $F$ and that

$$
\operatorname{Tr} T(F)=\int T_{F}(q, q) d Q .
$$

The previous results are collected in the following theorem:

Theorem 4.3. For every continuous $F \in \mathscr{H}, T(F)$ is a self-adjoint non negative trace class operator. In particular the transfer matrix $T$ is selfadjoint, trace class, non negative and such that $Z_{\Lambda}^{(P)}=\operatorname{Tr}\left(T^{2 L_{1}+1}\right)$.

$T$ is a positivity improving operator having $\|T\|$ as its highest eigenvalue; this eigenvalue is simple and the corresponding eigenvector can be taken to be positive.

A brief comment is in order here about the relation between the transfer matrix $T$ and the transfer matrix $T_{0}$ in the " $A_{0}=0$ " gauge $[11,14,15]$, with kernel

$$
T_{0}\left(q, q^{\prime}\right)=F(q) I_{0}\left(q, q^{\prime}\right) F\left(q^{\prime}\right),
$$

where

$$
\begin{aligned}
I_{0}\left(q, q^{\prime}\right)= & \exp \left\{-\frac{1}{4} \sum_{n \in \tilde{\Lambda}} P(\varrho(n))\right\} \times \exp \left\{\beta_{l} \sum_{n \in \tilde{\Lambda}} \varrho(n) \varrho^{\prime}(n) \cos \left(\theta(n)-\theta^{\prime}(n)\right)\right. \\
& \left.+\beta_{p} \sum_{n \in \tilde{\Lambda}} \cos \left(A(n, n+\underline{m})-A^{\prime}(n, n+\underline{m})\right)\right\} \\
& \cdot \exp \left\{-\frac{1}{4} \sum_{n \in \tilde{\Lambda}} P\left(\varrho^{\prime}(n)\right)\right\} .
\end{aligned}
$$

Due to the fact that periodic boundary conditions are best suited to the developments of next Section, we prefer to use $T$ instead of $T_{0}$ [it is in terms of $T$, not of $T_{0}$, that $Z_{A}^{(P)}$ can be expressed as $\left.\operatorname{Tr}\left(T^{2 L_{1}+1}\right)\right]$ in spite of the fact that $T_{0}$, being strictly positive (as can be seen from a slight improvement of Lemma 4.2 and of results of [11]), can lead to a well defined Hamiltonian formalism for the underlying quantum field theory. 
The connection between the two definitions is explicitly given by

$$
T=P T_{0} P,
$$

where $P$ is the projector on the gauge invariant subspace $\mathscr{H}_{\text {inv. }}$ of $\mathscr{H}$, namely the subspace of vectors invariant under the (unitary) transformations

$$
\psi(q) \rightarrow \psi_{\chi}(q)=\psi(\chi q)
$$

with

$$
\begin{aligned}
\chi \theta(n) & =\theta(n)+\chi(n) \\
\chi A\left(n, n^{\prime}\right) & =A\left(n, n^{\prime}\right)+\chi(n)-\chi\left(n^{\prime}\right) .
\end{aligned}
$$

As a concluding remark of this section, we wish to observe that the ground state of the Hamiltonian $H_{\tilde{\Lambda}}$ constructed from $T_{0}$ through the definition $T_{0}=\exp \left(-\varepsilon H_{\tilde{\Lambda}}\right)$ coincides with the eigenvector of $T$ belonging to the eigenvalue $\|T\|$.

Call $\Omega$ and $\Omega_{0}$, respectively, these vectors; by the Simon-Perron-Frobenius theorem both $\Omega$ and $\Omega_{0}$ can be taken to be strictly positive, and therefore are not orthogonal. Due to the uniqueness statement of the same theorem and to the fact that $T$ and $T_{0}$ commute with time independent gauge transformations both vectors must transform according to characters of the gauge group, but, as $T$ vanishes on $\mathscr{H} \ominus \mathscr{H}_{\text {inv. }}, \Omega$ must be in $\mathscr{H}_{\text {inv. }}$ and therefore $\Omega_{0}$, in order not to be orthogonal to $\Omega$, must itself transform according to the trivial character, namely it must belong to $\mathscr{H}_{\text {inv. }}$, so that $\left\|T_{0}\right\|=\|T\|$.

The equality of $\Omega$ and $\Omega_{0}$ follows then from the uniqueness part of the SimonPerron-Frobenius theorem.

For a discussion of the gauge invariance of the ground state in the Hamiltonian framework we refer to [12].

\section{Existence of the Thermodynamic Limit}

In this section we study the existence of the thermodynamic limit for the generating functional

$$
\langle\exp \{i[A(m)+\varphi(J)]\}\rangle,
$$

where

$$
A(m)=\sum_{n<n^{\prime}} m\left(n, n^{\prime}\right) A\left(n, n^{\prime}\right), m\left(n, n^{\prime}\right) \in \mathbb{Z} ; \quad \boldsymbol{\varphi}(\boldsymbol{J})=\sum_{n} \boldsymbol{J}(n) \cdot \boldsymbol{\varphi}(n), \boldsymbol{J}(n) \in \mathbb{R}^{2}
$$

and some of its properties.

Call

$$
\alpha_{\Lambda}^{(P)}(r)=|\Lambda|^{-1} \log \int d \sigma_{\Lambda} \exp \left\{-U_{\Lambda}^{(P)}+r \sum_{n \in \Lambda} \varrho(n)\right\}, \quad r \geqq 0,
$$

then the main estimate is:

\section{Lemma 5.1.}

$$
\left[Z_{A}^{(P)}\right]^{-1} \int d \sigma_{A} \exp \left\{-U_{A}^{(P)}\right\} \exp \left\{\sum_{n \in A} r(n) \varrho(n)\right\} \leqq \exp \left\{\sum_{n \in \Lambda}\left[\alpha_{\Lambda}^{(P)}(r(n))-\alpha_{\Lambda}^{(P)}(0)\right]\right\}
$$

moreover the limit $\alpha_{\infty}^{(P)}(r)=\lim _{\Lambda \rtimes \mathbb{Z}^{d}} \alpha_{\Lambda}^{(P)}(r)$ exists finite. 
Proof. We follow the methods of [3] to which we refer for more details. We must only prove that for $F_{1}, F_{2}$ continuous and square integrable $T\left(F_{1}, F_{2}\right)$ is trace class with

$$
\operatorname{Tr}\left(\left|T\left(F_{1}, F_{2}\right)\right|\right) \leqq\left[\operatorname{Tr}\left(T\left(F_{1}\right)\right)\right]^{1 / 2}\left[\operatorname{Tr}\left(T\left(F_{2}\right)\right)\right]^{1 / 2}
$$

and that

$$
\left|\operatorname{Tr}\left(\prod_{J=-L}^{L} T\left(F_{J}, F_{J+1}\right)\right)\right| \leqq \prod_{J=-L}^{L}\left(\operatorname{Tr}\left[T\left(F_{J}\right)^{2 L+1}\right]\right)^{\frac{1}{2 L+1}}
$$

for $F_{J}$ continuous, square integrable and always different from zero. We know that $T\left(F_{J}\right)$ is a trace class non negative selfadjoint operator with

$$
\operatorname{Tr}\left(T\left(F_{J}\right)\right) \leqq \text { const }\left\|F_{J}\right\|_{2}^{2}
$$

and the first result is a consequence of the elementary inequality

$$
\left|\left\langle\psi, T\left(F_{1}, F_{2}\right) \phi\right\rangle\right| \leqq\left\langle\psi, T\left(F_{1}\right) \psi\right\rangle^{1 / 2}\left\langle\phi, T\left(F_{2}\right) \phi\right\rangle^{1 / 2}
$$

because the operator $I$ is a non negative bounded selfadjoint operator and for $\psi$ and $\phi$ of compact support $\left\langle\psi, T\left(F_{1}, F_{2}\right) \phi\right\rangle=\left\langle F_{1} \psi, I F_{2} \phi\right\rangle$ so we can use Schwarz inequality and, by a limiting argument, prove the previous inequality for any vectors $\psi$ and $\phi$.

From Hölder's inequality for traces we get

$$
\left|\operatorname{Tr}\left(\prod_{J=-L}^{L} T\left(F_{J}, F_{J+1}\right)\right)\right| \leqq \prod_{J=-L}^{L}\left[\operatorname{Tr}\left(\left|T\left(F_{J}, F_{J+1}\right)\right|^{2 L+1}\right)\right]^{\frac{1}{2 L+1}} .
$$

Now, call

$$
A=\left|T\left(F_{J}, F_{J+1}\right)\right|, \quad T\left(F_{J}, F_{J+1}\right)=W A, \quad B=W^{*} T\left(F_{J}\right) W, \quad C=T\left(F_{J+1}\right)
$$

then, again by using Hölder's inequality, we get

$$
\operatorname{Tr}\left(A^{2 L+1}\right) \leqq\left[\operatorname{Tr}\left(B^{2 L+1}\right)\right]^{1 / 2}\left[\operatorname{Tr}\left(C^{2 L+1}\right)\right]^{1 / 2} .
$$

As $F_{J+1}$ is continuous and nowhere vanishing, $\frac{1}{F_{J+1}}$ exists continuous and if $f_{n}$ is a sequence of square integrable functions of compact support which is convergent to $f \in \mathscr{H}$ we have

$$
\left(T\left(F_{J}\right) f_{n}\right)(q)=\int \bar{F}_{J}(q) I\left(q, q^{\prime}\right) F_{J+1}\left(q^{\prime}\right)\left[\frac{F_{J}\left(q^{\prime}\right)}{F_{J+1}\left(q^{\prime}\right)} f_{n}\left(q^{\prime}\right)\right]=\left(T\left(F_{J}, F_{J+1}\right)\left(\frac{F_{J}}{F_{J+1}} f_{n}\right)\right)(q)
$$

This means that $\operatorname{Ran} T\left(F_{J}\right) \subseteq \overline{\operatorname{Ran} T\left(F_{J}, F_{J+1}\right)}$ from which it follows that $W W^{*} T\left(F_{J}\right)=T\left(F_{J}\right)$ because $W W^{*}$ is the projection on $\overline{\operatorname{Ran} T\left(F_{J}, F_{J+1}\right)}$. Now $\operatorname{Tr}\left(W^{*} T\left(F_{J}\right) W W^{*} \ldots W W^{*} T\left(F_{J}\right) W\right)=\operatorname{Tr}\left(W W^{*} T\left(F_{J}\right)^{2 L+1}\right) \leqq \operatorname{Tr}\left(T\left(F_{J}\right)^{2 L+1}\right)$ so we get the required inequality.

The first part of Lemma 5.1 follows from the inequalities just established by the same methods as in [3]; moreover $\alpha_{\Lambda}^{(P)}(r)$ is decreasing in $\Lambda$ and non negative by GKS inequalities, so the thermodynamic limit $\alpha_{\infty}^{(P)}(r)$ exists finite. 
Now we focus our attention on some correlation functions. First of all we have for $\theta(p)=\sum_{n} p(n) \theta(n), p(n) \in \mathbb{Z}:$

Lemma 5.2. For free boundary conditions the limit

$$
\lim _{\Lambda \pi \mathbb{Z}^{d}}\left\langle\exp \{i A(m)\} \exp \{i \theta(p)\} \varrho\left(n_{1}\right) \ldots \varrho\left(n_{K}\right)\right\rangle_{\Lambda}
$$

exists finite and is reached by monotone increase.

Proof. First of all

$$
\left\langle\exp \{i A(m)\} \exp \{i \theta(p)\} \varrho\left(n_{1}\right) \ldots \varrho\left(n_{K}\right)\right\rangle_{\Lambda}=\left\langle\cos (A(m)+\theta(P)) \varrho\left(n_{1}\right) \ldots \varrho\left(n_{K}\right)\right\rangle_{\Lambda}
$$

and monotone increase follows from GKS inequalities. From GKS inequalities it follows also that

$$
\begin{aligned}
& \left\langle\exp \{i A(m)\} \exp \{i \theta(p)\} \varrho\left(n_{1}\right) \ldots \varrho\left(n_{K}\right)\right\rangle_{\Lambda} \leqq\left\langle\varrho\left(n_{1}\right) \ldots \varrho\left(n_{K}\right)\right\rangle_{\Lambda}^{(P)} \\
& \leqq\left\langle\exp \left\{\sum_{n} r(n) \varrho(n)\right\}\right\rangle_{\Lambda}^{(P)}
\end{aligned}
$$

for a suitable field $r$ of compact support and non negative. Lemma 5.1 provides then the upper bound which completes the proof.

As the correlation functions $\left\langle\exp \left\{i A\left(n_{1}, n_{1}^{\prime}\right)\right\} \ldots \exp \left\{i A\left(n_{h}, n_{h}^{\prime}\right)\right\} \varphi_{i_{1}}\left(n_{h+1}\right)\right.$ $\left.\ldots \varphi_{i_{K}}\left(n_{h+K}\right)\right\rangle_{\Lambda}$

$$
i_{p} \in\{1,2\}, \quad n_{1}, \ldots, n_{h+K}, \quad n_{1}^{\prime}, \ldots, n_{h}^{\prime} \in \Lambda
$$

are linear combinations of expectations of the type

$$
\left\langle\exp \{i A(m)\} \exp \{i \theta(p)\} \varrho\left(n_{h+1}\right) \ldots \varrho\left(n_{h+K}\right)\right\rangle_{\Lambda},
$$

there exists the thermodynamic limit

$$
\begin{aligned}
\lim _{\Lambda \rtimes \mathbb{Z}^{d}}\left\langle\exp \{i A(m)\} \varphi_{i_{1}}\left(n_{h+1}\right) \ldots \varphi_{i_{K}}\left(n_{h+K}\right)\right\rangle_{\Lambda} \\
\quad=\left\langle\exp \{i A(m)\} \varphi_{i_{1}}\left(n_{h+1}\right) \ldots \varphi_{i_{K}}\left(n_{h+{ }_{K}}\right)\right\rangle .
\end{aligned}
$$

Now define the generating functional in the finite volume $\Lambda$ by

$$
W_{\Lambda}(m, \boldsymbol{J})=\langle\exp \{i[A(m)+\boldsymbol{\varphi}(\boldsymbol{J})]\}\rangle_{\Lambda}
$$

with $m$ and $\boldsymbol{J}$ lattice fields with bounded support $M$ contained in $\Lambda$. Of course, for fixed $m, W_{\Lambda}(m, \boldsymbol{J})$ is an entire function of $\boldsymbol{J} \in \mathbb{C}^{2|M|}$. Our main result concerning the generating functional is:

Theorem 5.3. $W_{\infty}(m, \boldsymbol{J})=\lim _{\Lambda \rightarrow \mathbb{Z}^{d}} W_{\Lambda}(m, \boldsymbol{J})$ exists and is an entire analytic function of the complex variables $\boldsymbol{J}=\left\{J_{i}(n)\right\}_{\substack{i=1,2 \\ n \in M}} \in \mathbb{C}^{2|M|}$.

Proof. By Vitali's theorem [17] we must only prove that

$$
\sup _{\Lambda} \sup _{\mathbf{J} \in K}\left|W_{\Lambda}(m, \boldsymbol{J})\right|<+\infty
$$


for each compact set $K \subset \mathbb{C}^{2|M|}$. But

$$
\left|W_{A}(m, \boldsymbol{J})\right| \leqq\left\langle\exp \left\{\sum_{n \in M}\|\boldsymbol{J}(n)\| \varrho(n)\right\}\right\rangle_{\Lambda} \leqq\left\langle\exp \left\{\sum_{n \in M} \mathrm{r}(\mathrm{n}) \varrho(\mathrm{n})\right\}\right\rangle_{\Lambda}^{(P)}
$$

with $r(n)=\sup _{\boldsymbol{J} \in \boldsymbol{K}}\|\boldsymbol{J}(n)\|$ which is uniformly bounded in $\Lambda$ by Lemma 5.1.

Obviously $W_{\infty}(m, \boldsymbol{J})$ for $\boldsymbol{J} \in \mathbb{R}^{2|M|}$ is a positive definite function on the dual group of $T^{L(M)} \times \mathbb{R}^{2|M|}$ so, by Bochner's theorem there exists a probability measure $\mu_{M}$ on $T^{L(M)} \times \mathbb{R}^{2|M|}$ such that

$$
W_{\infty}(m, \boldsymbol{J})=\int d \mu_{M} \exp \{i[A(m)+\boldsymbol{\varphi}(\boldsymbol{J})]\} .
$$

We can go even further and prove that for every continuous bounded function $f$ of the local configurations in $M$ there exists the thermodynamic $\operatorname{limit} \lim _{\Lambda \rightarrow \mathbb{Z}^{d}}\langle f\rangle_{\Lambda}=\langle f\rangle$ and that $\langle f\rangle=\int d \mu_{M} f$, so the collection of measures $\left\{\mu_{M}\right\}_{M \subset \mathbb{Z}^{d}}$ will define a state on the $C^{*}$-algebra $\mathscr{A}=\overline{\bigcup_{M} \mathscr{A}_{M}}$ where $\mathscr{A}_{M}$ is the $C^{*}$-algebra of continuous bounded functions of the local configurations $\{\varphi, A\}$ in $M$; this state is obviously translationally and gauge invariant. First of all we give the following useful bound:

Lemma 5.4. Define the probability measures $\mu_{M}^{\Lambda}$ by $\mu_{M}^{\Lambda}(f)=\langle f\rangle_{\Lambda} \Lambda \supset M$ for any $f \in \mathscr{A}_{M}$; then the measures $\mu_{M}^{\Lambda}$ are bounded, uniformly in $\Lambda$, by a fixed measure $v_{M}$ of finite total mass.

Proof. For $f \geqq 0$

$$
\begin{aligned}
& \langle f\rangle_{\Lambda}=Z_{\Lambda}^{-1} \int d \sigma_{\Lambda} \exp \left\{-U_{\Lambda}\right\} f \\
& \leqq Z_{M}^{-1} \int d \sigma_{M} \exp \left\{-U_{M}\right\} f\left[Z_{\Lambda \backslash M}^{-1} \int d \sigma_{\Lambda \backslash M} \exp \left\{-U_{\Lambda \backslash M}\right\} \exp \left\{\beta_{p} n_{\partial M}\right\}\right. \\
& \left.\cdot \exp \left\{\beta_{l} \sum_{n, n^{\prime}} \varrho(n) \varrho\left(n^{\prime}\right)\right\}\right]
\end{aligned}
$$

(by GKS inequalities), where $n_{\partial M}$ is the number of plaquettes around the boundary of $M$ and in the sum $\sum_{n, n^{\prime}} \varrho(n) \varrho\left(n^{\prime}\right) n \in \partial M$ and $n^{\prime} \in \Lambda \backslash M$ with $\left|n-n^{\prime}\right|=1$. Now $\exp \left\{\beta_{l} \sum_{n, n^{\prime}} \varrho(n) \varrho\left(n^{\prime}\right)\right\}=\exp \left\{\sum_{n^{\prime} \in \Lambda \backslash M} r\left(n^{\prime}\right) \varrho\left(n^{\prime}\right)\right\}$ for a suitable lattice field $r\left(n^{\prime}\right) \geqq 0$ and by GKS inequalities and the estimate of Lemma 5.1

$$
Z_{\Lambda \backslash M}^{-1} \int d \sigma_{\Lambda \backslash M} \exp \left\{-U_{\Lambda \backslash M}\right\} \exp \left\{\sum_{n \in \Lambda \backslash M} r(n) \varrho(n)\right\} \leqq \exp \left\{\sum_{n \in B(M)} \alpha_{\Lambda}^{(P)}(r(n))\right\},
$$

where $B(M)$ is the set of points of $\Lambda \backslash M$ which are nearest neighbors of points in $\partial M$. Using the elementary estimate $\alpha_{\Lambda}^{(P)}(r) \leqq A+K r^{4 / 3}$ with suitable positive constants $A$ and $K$, we see that the function

$$
\begin{aligned}
& \varphi_{\Lambda}\left(\{\varrho(n)\}_{n \in M}\right) \\
& \quad=\exp \left\{\beta_{p} n_{\partial M}\right\} Z_{\Lambda \backslash M}^{-1} \int d \sigma_{\Lambda \backslash M} \exp \left\{-U_{\Lambda \backslash M}\right\} \exp \left\{\beta_{l} \sum_{n, n^{\prime}} \varrho(n) \varrho\left(n^{\prime}\right)\right\}
\end{aligned}
$$

is bounded, uniformly in $\Lambda$, by a function $\varphi\left(\{\varrho(n)\}_{n \in M}\right)$ summable with respect to the measure $Z_{M}^{-1} \exp \left\{-U_{M}\right\} d \sigma_{M}$. 
In the following we will use the symbols $P$ and $Q$ in order to denote trigonometrical polynomials $P(\varphi)=\sum_{K} c_{K} \exp \left\{i \varphi\left(\boldsymbol{J}_{K}\right)\right\}, Q=\sum_{K} c_{K} \exp \left\{i A\left(m_{K}\right)\right\}$ of variables $\{\varphi(n)\}_{n \in M} \in \mathbb{R}^{2|M|}$ and $\left\{A\left(n, n^{\prime}\right)\right\}_{n, n^{\prime} \in M} \in T^{L(M)}$.

We know from Theorem 5.3 that there exists the thermodynamic limit $\lim _{A \rightarrow \mathbb{Z}^{d}}\langle P Q\rangle_{A}=\int d \mu_{M} P Q$. Now, for $f \in \mathscr{S}\left(\mathbb{R}^{2|M|}\right)$ there exists a family $\left\{P_{i}\right\}$ of trigonometrical polynomials with the following properties:

i) There exists $a>0$ such that $\left\|P_{i}\right\|_{\infty} \leqq a \forall i$.

ii) For every compact set $K \subset \mathbb{R}^{2|M|}$ and for every $\varepsilon>0$ there exists $P_{i}$ such that

$$
\left|f(x)-P_{i}(x)\right|<\varepsilon \forall x \in K
$$

$\mathrm{SO}$

$$
\begin{aligned}
\mid\langle f Q\rangle_{A} & -\int d \mu_{M} f Q \mid \leqq\|Q\|_{\infty}\left\langle\left|f-P_{i}\right| \chi_{K}\right\rangle_{A}+\|Q\|_{\infty}\left\|f-P_{i}\right\|_{\infty}\left\langle\chi_{C K}\right\rangle_{A} \\
& +\|Q\|_{\infty} \int d \mu_{M}\left|f-P_{i}\right| \chi_{K}+\|Q\|_{\infty}\left\|f-P_{i}\right\|_{\infty} \mu_{M}(C K)+\mid\left\langle P_{i} Q\right\rangle_{A} \\
& -\int d \mu_{M} P_{i} Q \mid
\end{aligned}
$$

for every $P_{i}$ and every compact $K$. Now by Lemma 5.4 we choose $K$ such that

$$
\|Q\|_{\infty}\left\langle\chi_{C K}\right\rangle_{A}+\|Q\|_{\infty}\left\|f-P_{i}\right\|_{\infty} \mu_{M}(C K)<\frac{\varepsilon}{3}
$$

for every $\Lambda$ and $P_{i}$, after which we can choose $P_{i}$ such that

$$
\|Q\|_{\infty}\left\langle\left|f-P_{i}\right| \chi_{K}\right\rangle_{A}+\|Q\|_{\infty} \int d \mu_{M}\left|f-P_{i}\right| \chi_{K}<\frac{\varepsilon}{3}
$$

uniformly in $\Lambda$ and by choosing $\Lambda$ large enough, in order that $\left|\left\langle P_{i} Q\right\rangle_{A}-\int d \mu_{M} P_{i} Q\right|<\frac{\varepsilon}{3}$, we have $\left|\langle f Q\rangle_{A}-\int d \mu_{M} f Q\right|<\varepsilon$. Now every $g \in C^{0}\left(T^{L(M)}\right)$ can, by the Stone-Weierstrass theorem, be uniformly approximated by trigonometrical polynomials $Q$ and, by the inequality

$$
\left|\langle f g\rangle_{A}-\int d \mu_{M} f g\right| \leqq 2\|f\|_{\infty}\|g-Q\|_{\infty}+\left|\langle f Q\rangle_{A}-\int d \mu_{M} f Q\right|
$$

we see that $\lim _{\Lambda \rightarrow \mathbb{Z}^{d}}\langle f g\rangle_{\Lambda}=\int d \mu_{M} f g$.

But every continuous function $\varphi$ of compact support in $\mathbb{R}^{2|M|}$ can be uniformly approximated by functions $f$ in $\mathscr{S}\left(\mathbb{R}^{2|M|}\right)$, so the same argument proves that, for any such $\varphi, \lim _{\Lambda \rightarrow \mathbb{Z}^{d}}\langle\varphi g\rangle \Lambda=\int d \mu_{M} \varphi g$.

Now we want to remove the restriction to observables which factorize in the link and site variables. Let $K$ be a compact interval in $\mathbb{R}^{2|M|}, \chi_{K}$ its characteristic function, we want to prove that $\lim _{\Lambda \rightarrow \mathbb{Z}^{d}}\left\langle P Q \chi_{K}\right\rangle_{\Lambda}=\int d \mu_{M} P Q \chi_{K}$. Let $V$ be an open interval containing $K$; now, by Urysohn's lemma, we can find a continuous function $\psi$ of compact support contained in $V$ such that $0 \leqq \psi \leqq 1$ and $\psi(x)=1 \forall x \in K$. Then

$$
\begin{aligned}
& \left|\left\langle P Q \chi_{K}\right\rangle_{A}-\int d \mu_{M} P Q \chi_{K}\right| \\
& \leqq\left|\langle P Q \psi\rangle_{A}-\int d \mu_{M} P Q \psi\right|+\|P Q\|_{\infty}\left\langle\left|\psi-\chi_{K}\right|\right\rangle_{A}+\|P Q\|_{\infty} \int d \mu_{M}\left|\psi-\chi_{K}\right| .
\end{aligned}
$$

Given $\varepsilon>0$, by a suitable choice of $V$ and by Lemma 5.4 we can have $\|P Q\|_{\infty}\left(\left\langle\left|\psi-\chi_{K}\right|\right\rangle_{\Lambda}+\int d \mu_{M}\left|\psi-\chi_{K}\right|\right)<\frac{\varepsilon}{2} \forall \Lambda$; moreover for $\Lambda$ large enough $\mid\langle P Q \psi\rangle_{\Lambda}$ 
$-\int d \mu_{M} P Q \psi \mid<\frac{\varepsilon}{2}$, so $\left|\left\langle P Q \chi_{K}\right\rangle_{\Lambda}-\int d \mu_{M} P Q \chi_{K}\right|<\varepsilon$. Having shown that the thermodynamic limit exists for any $Z \chi_{K}$ with $Z=\sum_{l} P_{l} Q_{l}$, we can complete the argument in this way: let $f$ be any continuous bounded function on $\mathbb{R}^{2|M|} \times \mathrm{T}^{L(M)}$, then for $K$ large enough

$$
\begin{aligned}
\left|\langle f\rangle_{\Lambda}-\int d \mu_{M} f\right| & \leqq\left|\left\langle f \chi_{K}\right\rangle_{\Lambda}-\int f \chi_{K} d \mu_{M}\right|+\|f\|_{\infty}\left(\left\langle\chi_{C K}\right\rangle_{\Lambda}+\mu_{M}(C K)\right) \\
& <\frac{\varepsilon}{3}+\left|\left\langle f \chi_{K}\right\rangle_{\Lambda}-\int f \chi_{K} d \mu_{M}\right| .
\end{aligned}
$$

Now, on $K \times T^{L(M)}, f$ can be uniformly approximated by polynomials $Z$ so that:

$$
\left\langle|f-Z| \chi_{K}\right\rangle_{\Lambda}+\int d \mu_{M}|f-Z| \chi_{K}<\frac{\varepsilon}{3}
$$

and

$$
\left|\langle f\rangle_{\Lambda}-\int f d \mu_{M}\right| \leqq \frac{2}{3} \varepsilon+\left|\int Z \chi_{K} d \mu_{M}-\left\langle Z \chi_{K}\right\rangle_{\Lambda}\right| \text {. }
$$

We summarize this discussion in the following theorem:

Theorem 5.5. The measures $\mu_{M}^{A}$ weakly converge, for $\Lambda \rtimes \mathbb{Z}^{d}$ to the measure $\mu_{M}$, which is absolutely continuous with respect to $d \sigma_{M}$.

\section{References}

1. Balian, R., Drouffe,J.M., Itzykson,C.: Phys. Rev. D 10, 3376-3395 (1974)

2. De Angelis, G.F., de Falco,D.: Lettere Nuovo Cimento 18, 536-538 (1977)

3. Fröhlich,J.: Phase transitions, goldstone bosons, and topological superselection rules. Proceedings of the Internationale Universitätswochen für Kernphysik, Schladmig, February 1976

4. Ginibre,J.: Commun. Math. Phys. 16, 310-328 (1970)

5. Glimm,J., Jaffe, A., Spencer, T.: The particle structure of the weakly coupled $P(\varphi)_{2}$ model and other applications of high temperature expansions. In: Constructive quantum field theory (ed. G.Velo, A.S.Wightman) Berlin-Heidelberg-New York: Springer 1973

6. Glimm, J., Jaffe, A. : Phys. Letters 66 B, 67-69 (1977)

7. Glimm, J., Jaffe, A. : Instantons in a $U(1)$ lattice gauge theory : a coulomb dipole gas. Preprint (1977)

8. Gohberg,I.C., Krein,M.G.: Introduction to the theory of nonselfadjoint operators. Providence: American Mathematical Society 1969

9. Guerra,F., Rosen,L., Simon, B.: Ann. Math. 101, 111-259 (1975)

10. Kogut,J.B.: Three lectures on lattice gauge theory. Lecture series presented at the International Summer Institute for Theoretical Physics. Bielefeld August 1976

11. Lüscher, M.: Construction of a selfadjoint strictly positive transfer matrix for Euclidean lattice gauge theories. Preprint (1976)

12. Lüscher, M.: Absence of spontaneous gauge symmetry breaking in Hamiltonian lattice gauge theories. Preprint (1977)

13. Nelson, E. : Probability theory and Euclidean field theory. In : Constructive quantum field theory (ed. G.Velo, A.S.Wightman). Berlin-Heidelberg-New York: 1973

14. Osterwalder, K.: Gauge theories on the lattice. Lecture delivered at the 1976 Cargèse Summer School. Preprint (1976)

15. Osterwald,K., Seiler,E.: Gauge field theories on the lattice. Preprint (1977)

16. Polyakov, A.M. : Nucl. Phys. B 120, $429-458$ (1977)

17. Simon, B.: The $P(\varphi)_{2}$ Euclidean (quantum) field theory. Princeton:Princeton University Press 1974

18. Wilson, K.G. : Phys. Rev. D 10, 2445-2459 (1974)

19. Yang,C.N., Mills, R.L.: Phys. Rev. 96, 191 (1954)

Communicated by A. Jaffe

Received May 31, 1977 